\title{
Viscometric analysis of the hydration of a surface cross- linked EM-HEC polymer
}

\author{
Nesani Premathasan and Spencer E. Taylor* \\ Department of Chemistry and Centre for Petroleum and Surface Chemistry, University of \\ Surrey, Guildford, Surrey GU2 7XH, UK
}

\begin{abstract}
Hydrophilic polymers are used in many different products and processes, in which they function, for example, as rheology control agents, steric stabilizers, water retention agents, or flocculants. When the application requires dissolution of polymer powders in water, this occurs through hydration of the hydrophilic chains, and is accompanied by an increase in solution viscosity through a combination of chain entanglement, hydrophobic interactions and hydrogen bonding. On a practical level, however, the addition of high molecular weight polymer powder to water can result in the formation of a surface gel layer leading to partial hydration and particle agglomeration, creating operational and formulation problems. One way of overcoming this is by chemically crosslinking hydroxyl groups on the particle surfaces, for example, by reaction with dialdehydes, such as glyoxal. This retards the initial hydration rate, thereby allowing more time for effective powder dispersion. The present study is concerned with factors affecting the hydration of a commercial non-ionic glyoxal-crosslinked methyl ethyl hydroxyethylcellulose, in the concentration range $0.15-0.5 \mathrm{wt} \%$. Hydration kinetics have
\end{abstract}


been determined by monitoring the increase in solution viscosity with time using low shear rotational viscometry. Experimental variables include polymer concentration, temperature, $\mathrm{pH}$, the presence of anionic, cationic and non-ionic surfactants, and ionic strength (sodium chloride concentrations in the range $0-5 \mathrm{~mol} / \mathrm{L}$ ). In deionised water, the hydration process is shown to involve two kinetic steps, assigned to breakage of hemiacetal crosslinks and polymer hydration. The kinetic studies enabled the various stages in the overall hydration process to be analysed, and mechanistic implications and activation parameters to be assigned.

\section{Introduction}

Water-soluble polymers based on cellulose are used in many processes and products, where they serve different functions, including as rheology control agents, binders, emulsion stabilizers, and water retention agents [1]. In order to fulfil most of these functions, it is necessary to dissolve the polymer powders in water. Dissolution occurs by hydration of the hydrophilic groups, which is accompanied by an increase in solution viscosity through a combination of chain entanglement, hydrophobic interactions and hydrogen bonding. On a practical level, however, addition of the polymer powder to water can often be problematic if the hydration process begins before the powder has been adequately dispersed; under these conditions incomplete dissolution and particle agglomeration (lump formation) can be unwanted consequences. These problems, and possible means by which they can be overcome, have largely been confined to the patent literature for around 50 years, with Sharma et al. recently summarising the various mitigation approaches [2].

In the present study, we have conducted a detailed examination of one of the approaches used to overcome this problem which is based on surface crosslinking of the powdered polymers by suspending the powder in a polar organic liquid, such as ethanol or acetone, in the presence of low concentrations of formaldehyde or dialdehydes (e.g., glutaraldehyde or 
glyoxal) $[3,4]$. Reaction with the aldehydes reduces the availability of surface hydroxyl groups on cellulose ether particles (as in the original invention [3]) or synthetic polymers such as polyvinyl alcohol [5]. This modifies the particle wettability which delays hydration [6], thereby prolonging the dispersion time in water [7]. The resultant effect is that more effective dispersion of the particles can occur before the formation of sticky surface gel layers which are responsible for the unwanted effects described above [2,7].

Specifically, we have chosen to study the hydration of a glyoxal-treated non-ionic cellulose ether, commercially available as Bermocoll EM 7000 FQ from AkzoNobel. This product has applications, for example, in the formulation of water-based latex paints, where it acts as a rheology control agent and steric stabilizer to improve ease of application and drying behaviour [8]. In this particular application it is necessary that the product must be free from gel-lumps, and therefore it is essential that hydration is complete during formulation. According to the manufacturer, this polymer is based on a high viscosity grade of ethyl methyl hydroxyethylcellulose (EMHEC). As shown in Fig. 1(a), the general structure comprises hydroxyethylcellulose (HEC) backbones which have been treated with a mixture of methyl and ethyl chlorides to increase the overall hydrophobicity of the polymer (and therefore increase its potential for hydrophobic bonding). Subsequent treatment with glyoxal results in a degree of surface crosslinking via hemiacetal bonds, as shown in Fig. 1(b) [9]. Our interest in this study is to examine the hydration process of this polymer in aqueous solution, and identify how this is affected by variables such as polymer concentration, temperature, $\mathrm{pH}$, the presence of different surfactants, and ionic strength $(\mathrm{NaCl})$. To the best of our knowledge, this is the most detailed study of the hydration of this type of polymer. 


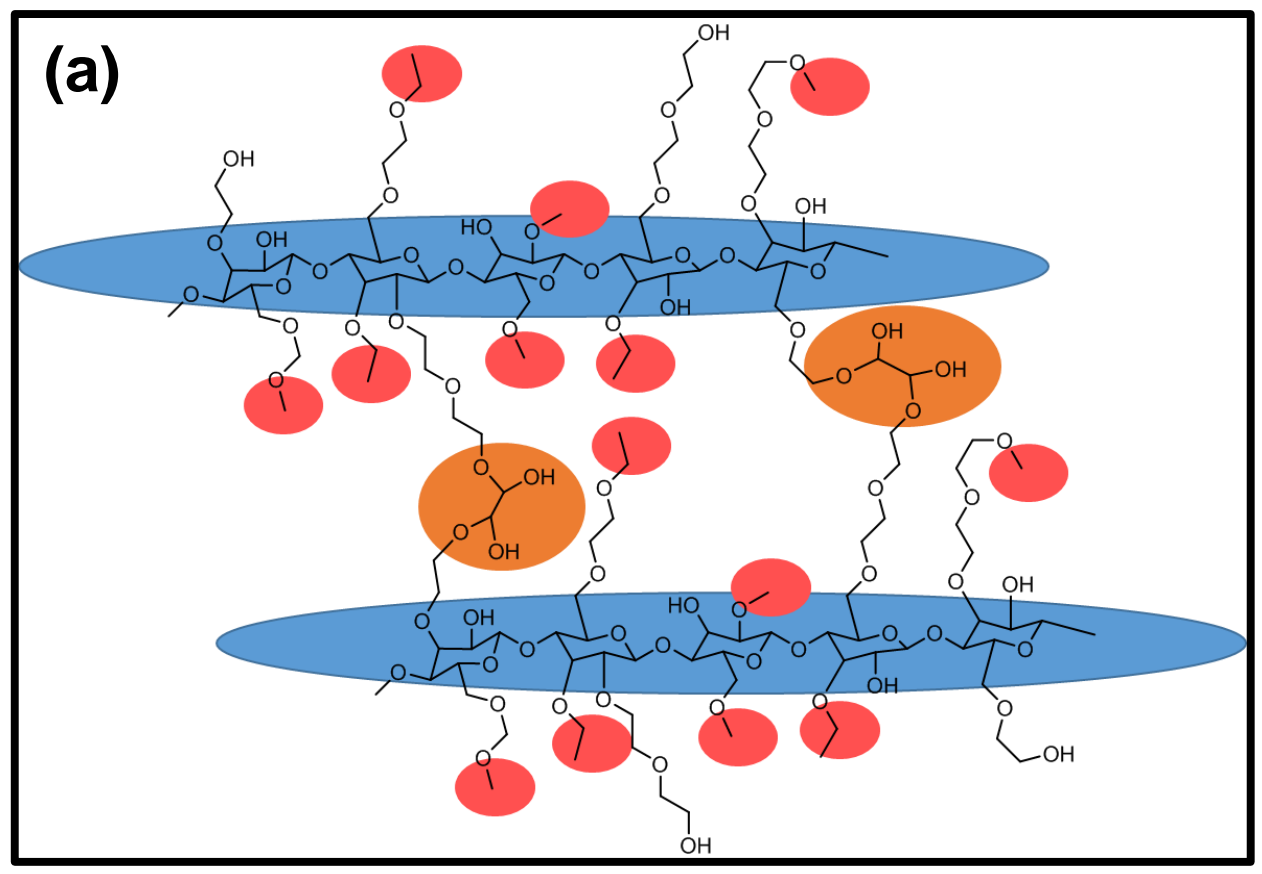

(b)

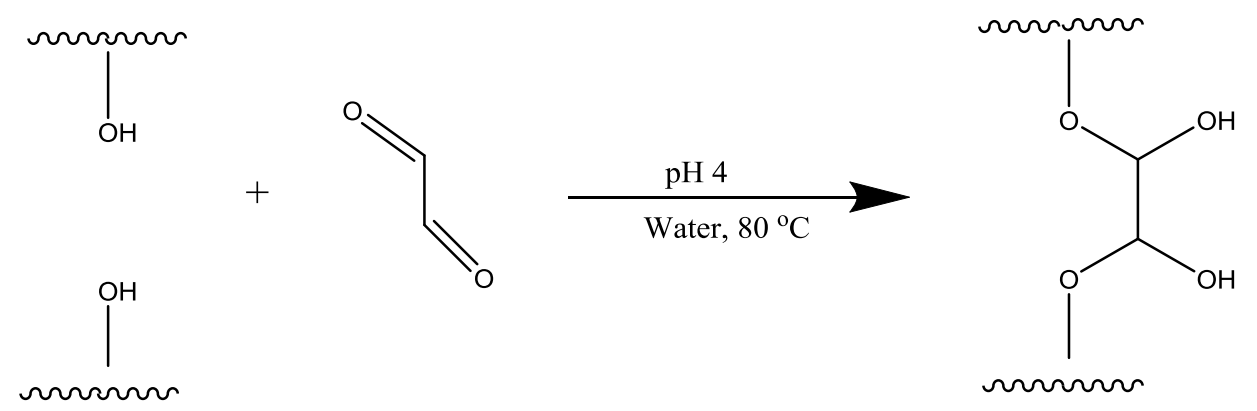

Fig. 1. (a) Partial structure of Bermocoll EM 7000 FQ: hydrophilic cellulose backbone (blue), hydrophobic ethyl and methyl groups (red), and glyoxyl bridges (orange). (b) Glyoxal crosslinking reaction [9].

\section{Experimental}

\subsection{Materials}

Bermocoll EM 7000 FQ, a glyoxal-crosslinked version of a high viscosity grade of EMHEC, was obtained from AkzoNobel Functional Chemicals AB, Stenungsund, Sweden. According to the manufacturer [8], the average degree of polymerization $\left(D_{p}\right)$ is 7000 , with degrees of methyl and ethyl substitution ( $D S_{M e}$ and $D S_{E t}$, respectively) of 0.4-0.5 and molar substitution of ethylene oxide $\left(M S_{E O}\right)$ of 1.6-1.8, leading to a calculated molecular weight [given by $D_{p} \times$ 
$\left.\left(162+44 M S_{E O}+28 D S_{E t}+14 D S_{M e}\right)\right]$ of $\sim 2 \times 10^{6} \mathrm{~g} \mathrm{~mol}^{-1}$. Sodium chloride $(\mathrm{NaCl}$; for ionic strength control) and sodium hydroxide ( $\mathrm{NaOH}$; for $\mathrm{pH}$ control) were from Acros Organics (Fisher Scientific, Loughborough, UK). The surfactants sodium dodecyl sulfate (SDS), cetyltrimethylammonium bromide (CTAB), Triton X-100 (TX-100) were all purchased from Sigma-Aldrich Ltd (Poole, UK) and used as received. Water was deionised from a Millipore Direct-X system (resistivity $=18.2 \mathrm{M} \Omega \mathrm{cm})$.

\subsection{Methods}

Aqueous phases with the appropriate ionic strength (using $\mathrm{NaCl}$ ) and surfactant concentration were pre-equilibrated at the required temperature (in the range $25-50{ }^{\circ} \mathrm{C}$ ). When necessary, the $\mathrm{pH}$ was increased (with $\mathrm{NaOH}$ solution) at the temperature of the experiment. The temperature was found to be controllable to within $\pm 0.5^{\circ} \mathrm{C}$ of the desired value for the duration of each experiment by placing an inverted Petri dish under the beaker once the specific temperature had been reached through direct contact with the metal hotplate. The air-space created by the Petri dish acted as a fine control of the heat being applied to the beaker and its contents to maintain the constant temperature. Hydration experiments were carried out by adding weighed amounts of dry polymer powder to the requisite stirred aqueous phase (100 $\mathrm{mL}$ ) contained in a $250 \mathrm{~mL}$ glass beaker on a stirrer/hot-plate. The stirring speed was maintained at a mild-vortex level that enabled effective initial dispersion of the polymer powder without entraining air which could lead to the formation of polymer-stabilised bubbles. The polymer concentrations ranged from $0.15-0.5 \mathrm{wt} \%$. Hydration times depended on the conditions, the longest experiments taking approximately $3 \mathrm{~h}$.

Samples $(\sim 1.5 \mathrm{~mL})$ for viscometric analysis were removed by pipette at suitable time intervals during each experiment and were immediately transferred to the LS-1/1 measuring system of a Low Shear 30 viscometer (Contraves A.G., Zürich, Switzerland) which had been 
pre-equilibrated to the same temperature as the experiment. This instrument, which is now available as the ProRheo LS 300 (ProRheo GmbH, Althengstett, Germany), is a rotational viscometer using the Couette principle (bob and cup geometry) and is especially useful for low viscosity liquids [10]. Apparent viscosity-shear rate flow curves were determined for each sample.

\section{Results and discussion}

Several different experimental variables are considered separately in the following sections for their effects on polymer hydration, monitored by the increase in viscosity with time. As will be seen, the behaviour of the kinetic curves follows a similar pattern throughout, which allowed a general kinetic model to be formulated.

\subsection{Effect of polymer concentration on hydration rate}

As shown in Fig. 2, during the dissolution process polymer hydration is accompanied by a change in solution rheology. Newtonian behaviour immediately following powder dispersion, which became increasingly shear thinning as hydration progressed. Although the viscometer is restricted in terms of the shear rate $(j)$ range available $\left(\sim 0.02-128 \mathrm{~s}^{-1}\right)$, the apparent viscosity, $\eta(\dot{\gamma})$, was found to be consistent with the Cross model, Eq. (1) [11]. In Eq. (1) $\eta_{0}$ and $\eta_{\infty}$ are the respective zero- and infinite-shear rate viscosities, $\dot{\gamma}_{c r i t}$ is the critical shear rate above which shear thinning becomes important, and $m$ is the Cross exponent which indicates the degree of shear thinning (for Newtonian liquids $m=0$, whereas maximum shear thinning occurs for $m=$ $1[12])$.

$$
\eta(\dot{\gamma})=\frac{\eta_{0}-\eta_{\infty}}{1+\left(\dot{\gamma}_{\dot{\gamma}_{\text {crit }}}\right)^{m}}+\eta_{\infty}
$$


Owing to the restricted high shear rate range attainable, we were unable to determine $\eta_{\infty}$ values directly or to extrapolate lower shear rate data unequivocally using Eq. (1). Instead, for the purposes of fitting the experimental points in Fig. 2, we therefore assumed that $\eta_{\infty}$ approximates to the aqueous viscosity $\left(0.70 \mathrm{mPa} \mathrm{s}\right.$ at $\left.35^{\circ} \mathrm{C}\right)$ at high shear rate, as previously considered elsewhere for solutions of guar gum and derivatives by Risica et al. [13]. In the present study this is considered to be a reasonable assumption, particularly at the lowest polymer concentrations corresponding to the semidilute regime (see later) where the high shear conditions would be most likely to break chain entanglements. In fact, the sensitivity of the other Cross equation parameters to the choice of realistic values of $\eta_{\infty}$ (i.e., up to $10 \mathrm{mPa}$ ) is sufficiently low as to result in minimal errors as a consequence; in support of this, applying unconstrained fitting of the apparent viscosity data for the most concentrated sample studied (0.5 wt $\%$ at 156 minutes) yielded $\eta_{\infty}=5.25 \mathrm{mPa}$.

One of the flow curves shown in Fig. 2 ( $0.25 \mathrm{wt} \%$ polymer at 60 minutes) illustrates a feature which was frequently observed throughout the study during the period immediately following the onset of hydration when sticky, gelled polymer particles are formed. These particles interfere with the viscometric measurements at low shear rate, as they bridge the measuring system geometry, thus producing erratic or irregularly high values.

The Cross model fitting parameters extracted from the data contained in Fig. 2 are summarised in Table 1 and are seen to be dependent on polymer concentration and hydration time (0.5 wt $\%$ data). It is evident that for the highest polymer concentration studied, $\eta_{0}$ increases as hydration proceeds, whereas both $\dot{\gamma}_{c r i t}$ and the Cross exponent decrease. 
Table 1. Values of the Cross model (Eq. (1)) fitting parameters for the hydration of Bermocoll EM $7000 \mathrm{FQ}$ in deionised water at $35^{\circ} \mathrm{C}$ for different polymer concentrations and hydration times.

\begin{tabular}{lllll}
\hline $\begin{array}{l}\text { Polymer } \\
\text { concentration (wt\%) }\end{array}$ & Hydration time (minutes) & $\eta_{0}(\mathrm{mPa} \mathrm{s})$ & $\dot{\gamma}_{\text {crit }}\left(\mathrm{s}^{-1}\right)$ & $m$ \\
\hline 0.15 & Average of 90, 135 and 180* & 7.37 & 1110 & 0.360 \\
0.25 & Average of 90,120 and 160* & 35.6 & 85.7 & 0.469 \\
0.50 & 96 & 45.7 & 20.2 & 0.634 \\
0.50 & 126 & 71.7 & 9.55 & 0.592 \\
0.50 & $156^{*}$ & 116 & 1.97 & 0.552 \\
\hline
\end{tabular}

*Represent fully hydrated values.
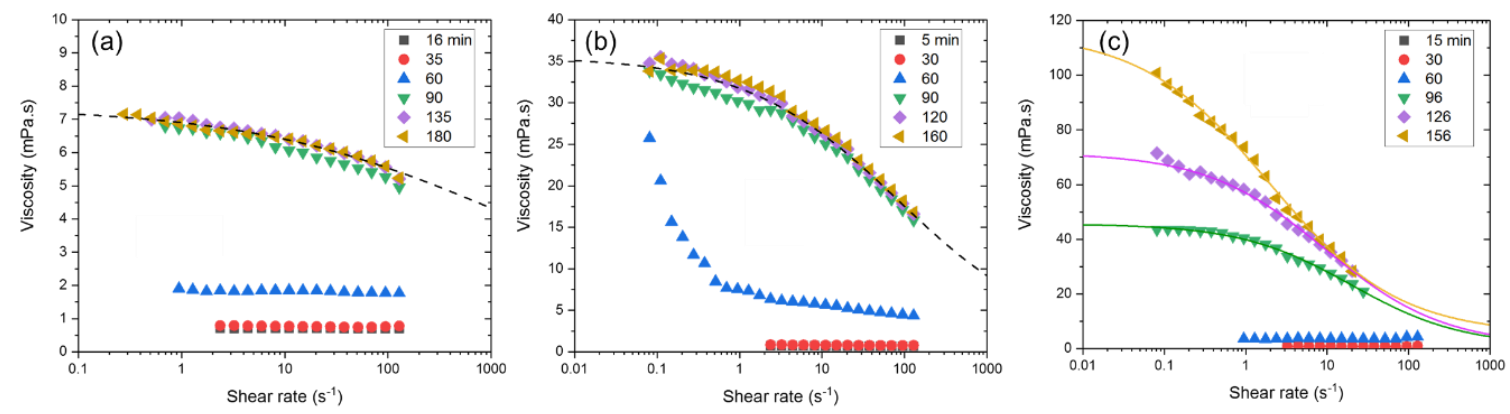

Fig. 2. Rheograms for EM $7000 \mathrm{FQ}$ hydration at $35^{\circ} \mathrm{C}$ for concentrations of (a) 0.15 , (b) 0.25 and (c) $0.5 \mathrm{wt} \%$ polymer in deionised water. Full lines represent Cross model fits to a single dataset. Black dashed lines in (a) and (b) represent average fits to more than one dataset. In (b), the low shear rate measurements made at 60 minutes are affected by the onset of hydration which causes some gelled particles to stick to the rheometer geometry; the blue dashed line is therefore the Cross model fit to higher shear rate data.

Fig. 3 shows the development of the zero-shear viscosity in the hydrating polymer solutions in deionised water. For each concentration it is seen that a certain time is required, in the region of $\sim 40$ minutes at $35^{\circ} \mathrm{C}$, before a significant increase in zero-shear viscosity becomes apparent; up to this point, the behaviour is Newtonian, as is also shown by the flow curves in Fig. 2, since the polymer is essentially insoluble at this stage. We interpret this initial "induction time" as 
the time required for hydrolysis of the crosslinking hemiacetal bonds, cleavage of which then allows subsequent hydration to take place, leading to the increase in apparent viscosity and the appearance of shear thinning rheology.

Thus, with increasing polymer concentration, increasing inter-molecular interactions, involving hydrogen bonding, hydrophobic bonding and chain entanglement, show up clearly in the $\eta_{0}$ values shown in Fig. 3(a). This is also evinced by the shear thinning behaviour in the flow curves shown in Fig. 2 following the onset of hydration. Where appropriate, for $0.15 \mathrm{wt} \%$ EM 7000 FQ solutions, $\eta_{0}$ values are subsequently quoted in this paper, based on average values taken in the low shear rate range (usually below $10 \mathrm{~s}^{-1}$ ).
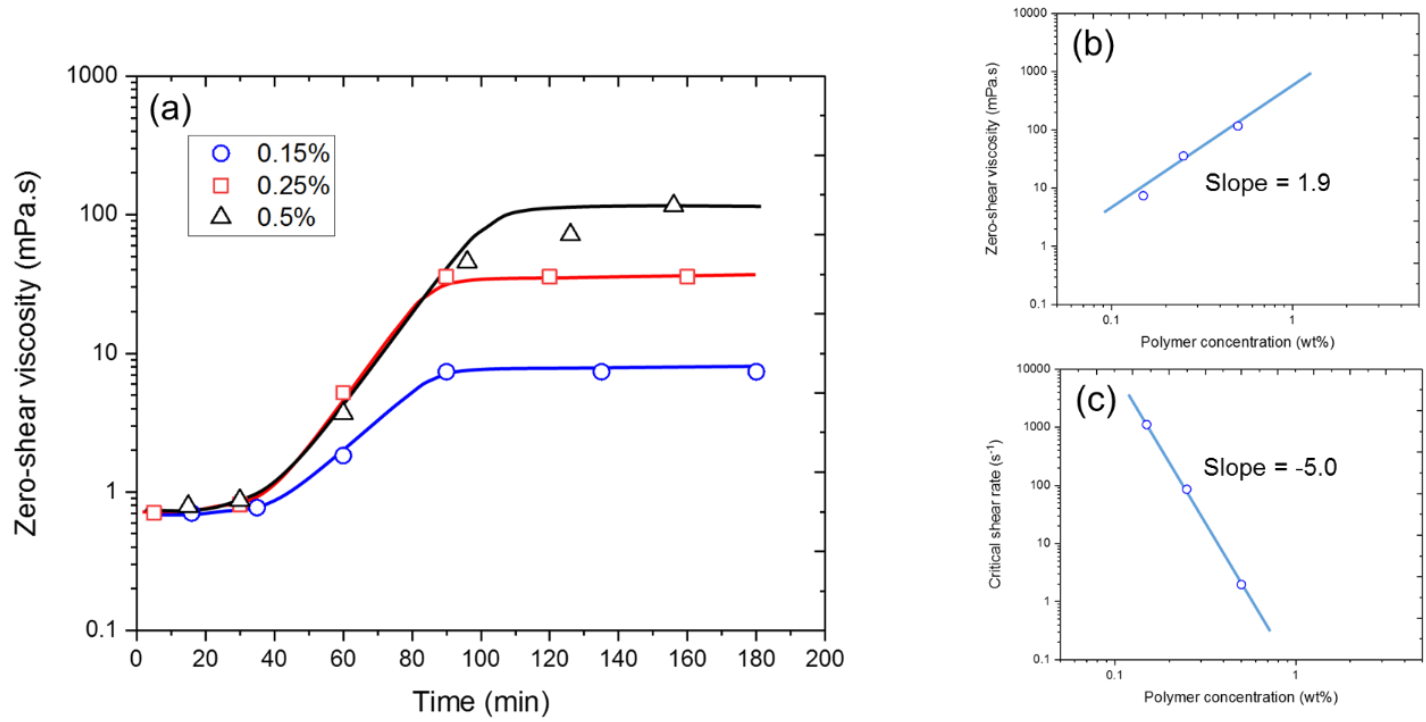

Fig. 3. (a) Time-dependence of the development of $\eta_{0}$ for different EM 7000 FQ concentrations at $35{ }^{\circ} \mathrm{C}$ in deionised water. (b) Double-logarithmic dependence of $\eta_{0}$ on polymer concentration. (c) Double-logarithmic dependence of $\dot{\gamma}_{\text {crit }}$ on polymer concentration.

The dependence of zero-shear viscosity on polymer concentration $\left(c_{p}\right)$ is given by the scaling relationship $\eta_{0} \propto c_{p}{ }^{1.9}$ evident from the double-logarithmic plot shown in Fig. 3(b). Over the years, polymer physicists have used scaling theory to provide insights into the extent of chain 
entanglement in polymer solutions [14,15]. Different scaling exponents are characteristic of different concentration/entanglement regimes. Dilute unentangled polymer solutions exhibit a low power-law scaling $(\sim 0)$ between viscosity and concentration, which increases as the concentration increases through semidilute unentangled, semidilute entangled and concentrated regimes $[13,16]$. Although the scaling factor of 1.9 found here should be considered to be approximate value owing to the limited data, together with the appearance of shear thinning, it is consistent with the hydrated polymer solutions being within the semidilute regime, possibly spanning unentangled and entangled regions [17]. This is consistent with an estimate of the concentration at which chains begin to overlap, $c^{*}$, being $\sim 0.1 \mathrm{wt} \%$ for this neutral polymer in water, using $c^{*} \approx D_{p}{ }^{(1-3 v)}[18]$, where $D_{p}$ is the degree of polymerisation (given in the Experimental section) and $v$ reflects the solvent quality and is 0.588 for neutral polymers in good solvents, i.e., $c^{*} \approx D_{p}{ }^{-0.764}$. The corresponding entanglement concentration, $c_{e}$, is given by $c_{e}=n^{(6 \mathrm{v}-2)} c^{*}[18]$, such that $c_{e}=n^{1.53} c^{*}$, where $n$ is the number of polymer chain overlaps which constitute an entanglement. Since this shows that $c_{e}>c^{*}$, our experimental concentration range is predicted to contain semidilute polymer solutions when fully dissolved.

In addition to the foregoing, the critical shear rate obtained from the Cross equation scales as $\dot{\gamma}_{c r i t} \propto c_{p}{ }^{-5.0}$, from Fig. 3(c), which reflects the sensitivity of the solution structure to polymer concentration and the increasing influence of chain entanglements within the concentration range studied.

\subsection{Effect of temperature on hydration rate}

The effect of temperature on hydration rate provides the framework for the development of a kinetic model for the dissolution of this polymer in deionised water. Fig. 4 shows the development of zero-shear viscosity at different temperatures for a constant $0.15 \mathrm{wt} \%$ EM 7000 FQ polymer. 


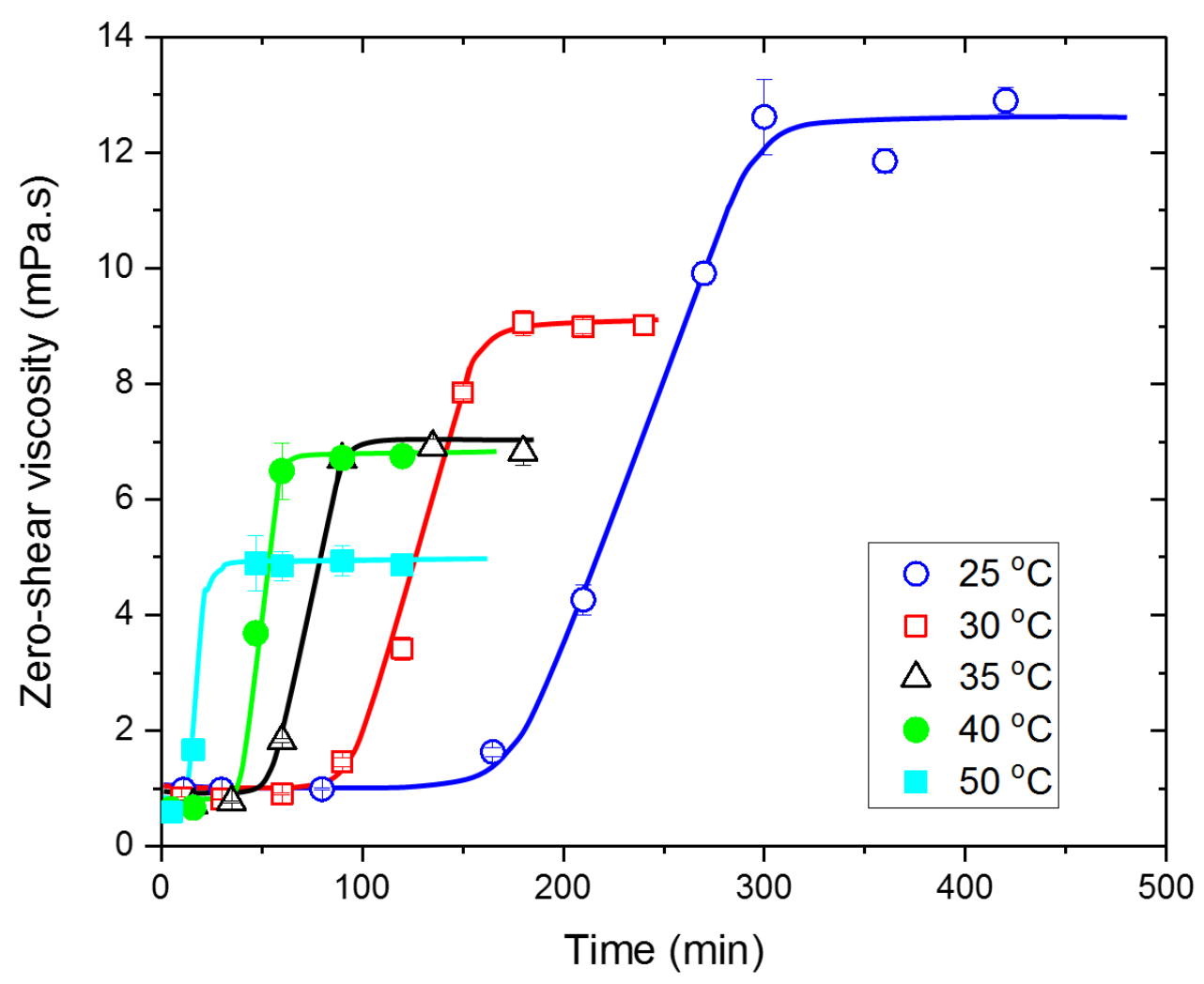

Fig. 4. The effect of temperature on the development of zero-shear viscosity during the hydration of $0.15 \mathrm{wt} \%$ EM 7000 FQ in deionised water. The curves are generated by fitting the data to Eq. (2).

Unsurprisingly, it is evident from Fig. 4 that temperature has a significant effect on the overall hydration process. It can be seen, for example, that increasing temperature results in more rapid hydration, but the lower final viscosity values reflect weakened interactions between the fully hydrated polymer molecules, i.e., lower gel-strength and hence lower viscosity. The sigmoidal shape of the kinetic curves also suggests that more than one process is involved. As mentioned in the previous section, it is apparent that the early stages involve an induction process, the extent of which decreases with increasing temperature. This is followed by an increase in solution viscosity. Based on this particular series of experiments, therefore, it appears that the induction process determines the overall hydration time, at least 
at the lower temperatures studied. As alluded to earlier, considering the nature of the polymer it is reasonable to assume that the induction step relates to the hydrolysis of the crosslinks, while the subsequent increase in solution viscosity is due to hydration of the polymer chains.

On this basis, the kinetic plots can be analysed according to Eq. (2) (see also the treatment given in [19]), in which $\eta_{0}(t)$ is the zero-shear viscosity at time $t, \eta_{0}(i)$ is the zero-shear viscosity of the initial aqueous solution, $\eta_{0}(f)$ is the final polymer solution zero-shear viscosity, $k$ is the first-order rate constant for hydration of the polymer chains, and $\tau_{\text {ind }}$ is the induction time for the breakdown of the hemiacetal crosslinks.

$$
\eta_{0}(t)=\eta_{0}(i)+\frac{\eta_{0}(f)-\eta_{0}(i)}{1+\exp \left[-k\left(t-\tau_{\text {ind }}\right)\right]}
$$

Eq. (2) has been fitted to the data in Fig. 4 using EasyPlot (Spiral Software), and the kinetic fitting parameters are summarised in Table 2.

Table 2. Kinetic fitting parameters (from Eq. (2)) for the hydration of $0.15 \mathrm{wt} \%$ Bermocoll EM 7000 FQ in deionised water at different temperatures.

\begin{tabular}{lllll}
\hline Temperature $\left({ }^{\circ} \mathrm{C}\right)$ & $\eta_{o}(i)(\mathrm{mPa} \mathrm{s})$ & $\eta_{o}(f)(\mathrm{mPa} \mathrm{s})$ & $\tau_{\text {ind }}(\mathrm{min})$ & $k\left(\mathrm{~min}^{-1}\right)$ \\
\hline 25 & 0.977 & 12.6 & 235 & 0.0397 \\
30 & 0.903 & 9.07 & 129 & 0.0822 \\
35 & 0.708 & 6.98 & 70.6 & 0.144 \\
40 & 0.650 & 6.73 & 47.0 & 0.245 \\
50 & 0.586 & 4.89 & 18.0 & 0.558 \\
\hline
\end{tabular}

The parameters in Table 2 indicate that $k$ increases with increasing temperature, with the induction time decreasing. If $1 / \tau_{\text {ind }}$ is taken as a measure of the rate of crosslink hydrolysis, Fig. 5 compares the rate behaviour of the two processes. 


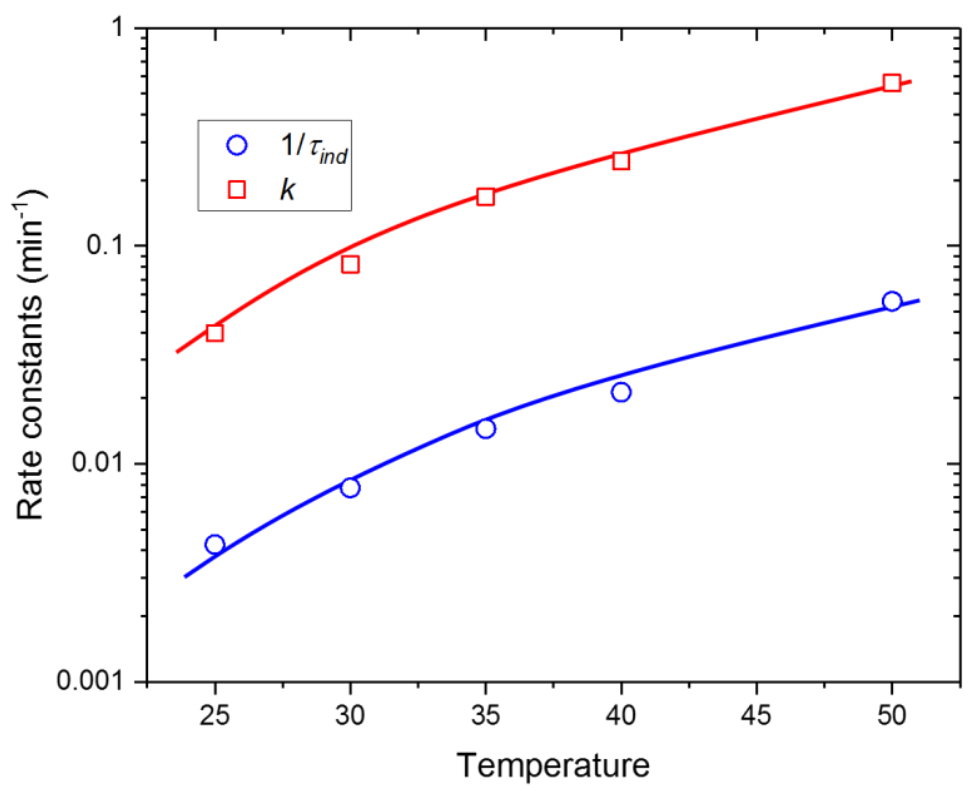

Fig. 5. The effect of temperature on the respective rates of hydration and induction (crosslink breakage) for $0.15 \mathrm{wt} \%$ EM 7000 FQ in deionised water. The lines are drawn to guide the eye.

It is seen from Fig. 5 that the rates of both processes increase steadily and uniformly over the temperature range studied. $k$ and $\tau_{\text {ind }}$ are plotted in Fig. 6(a) according to the Arrhenius equation,

$$
k \text { or } \tau_{\text {ind }}=A \exp \left(-\frac{E_{a}}{R T}\right)
$$

in which $A$ is the pre-exponential factor, $R$ is the gas constant, $T$ is the absolute temperature, and $E_{a}$ is the activation energy, the respective values of which are $83.1 \pm 6.3$ and $81.1 \pm 3.6$ $\mathrm{kJ} \mathrm{mol}^{-1}$, reflecting the similar origins of both processes, involving extensive bond making and breaking. For the hydration process, the slightly lower $E_{a}$ indicates that considerable rearrangement of the polymer structure is necessary before dissolution is complete, consistent with the breakage of intra-molecular hydrogen and hydrophobic bonds, and subsequent 
hydrogen bonding with water. Activation energies for simple diffusion-limited hydrophilic polymer dissolution in water are some $10 \times$ smaller than found here for the hydration rate; for example, $E_{a}$ for dissolution of alginate powder is $\sim 23 \mathrm{~kJ} / \mathrm{mol}$ [18], similar to that for the selfdiffusion of water (17.6 kJ/mol) [20]. On the other hand, the larger $E_{a}$ value for the induction process is consistent with making and breaking covalent chemical bonds.
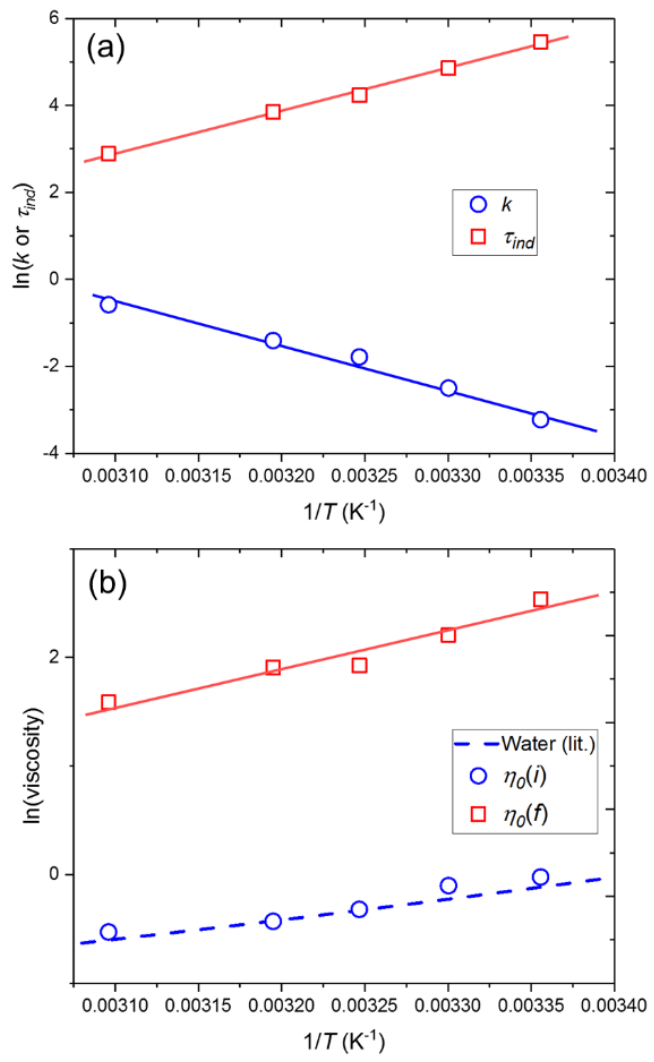

Fig. 6. (a) Arrhenius plots for induction time ( $\tau_{\text {ind }}$ in minutes $)$ and hydration rate constant $\left(k\right.$ in $\left.\min ^{-1}\right)$; and (b) Eyring plots for $\eta_{0}(i)$ and $\eta_{0}(f)$ (in $\mathrm{mPa}$ s) for $0.15 \mathrm{wt} \%$ EM $7000 \mathrm{FQ}$ in deionised water. Corresponding water viscosity data are also shown as the dashed line in (b).

In Fig. 6(b), are the corresponding Eyring [21] plots for the temperature dependence of $\eta_{0}(i)$ and $\eta_{0}(f)$ as well as literature viscosity data for water [22]. The activation energies associated with viscous flow are substantially lower than those involving chemical bonds, with the value for the initial viscosity, $16.0 \pm 2.1 \mathrm{~kJ} / \mathrm{mol}$, being very close to that of pure water $(15.2 \pm 0.2$ 
$\mathrm{kJ} / \mathrm{mol}$, calculated from data given by Kestin et al. [22]). The plot for the final viscosity containing dissolved polymer yields a higher activation energy of $28.7 \pm 4.3 \mathrm{~kJ} / \mathrm{mol}$, which reflects a stronger associating system consistent with more extensive chain entanglement involving intra-molecular and inter-molecular hydrogen bonds and hydrophobic interactions [9].

Fig. 7 shows the effects of temperature cycling on the polymer solutions that have either been prepared directly at the indicated temperatures, or prepared at the extreme temperatures in this range (i.e., 25 or $50^{\circ} \mathrm{C}$ ) and subsequently heated or cooled to the indicated temperatures. The reasonable consistency of the data, given of the thermal history of the solutions, not only demonstrates the reproducibility of the measurements, but also highlights the thermoreversibility of the fully hydrated polymer solutions within the (albeit small) temperature range studied.

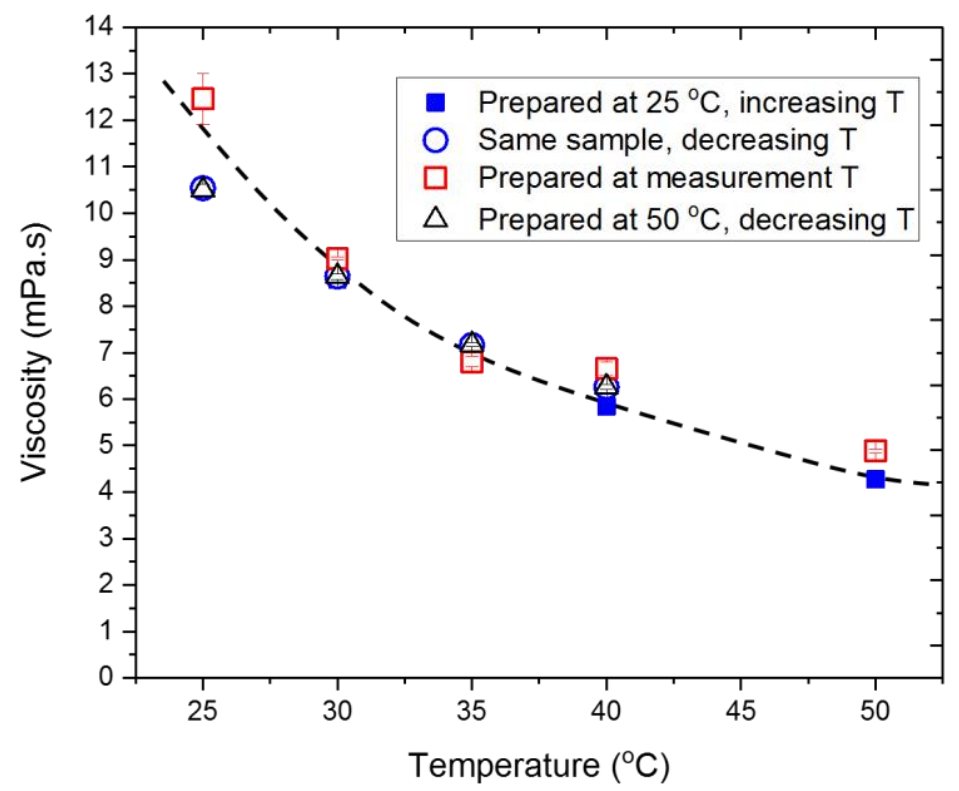

Fig. 7. Effect of temperature on the zero-shear viscosity of $0.15 \mathrm{wt} \%$ EM $7000 \mathrm{FQ}$ solutions hydrated at different temperatures. 


\subsection{Effect of $p H$ on hydration rate}

In aqueous solution, the hydrolysis of hemiacetal bonds can be both acid- and base-catalysed [23], and for this particular polymer the cleavage of the hemiacetal crosslinks is known to increase with temperature, as described above, or with $\mathrm{pH}[24]$.

Hydration reactions were carried out using deionised water which had been $\mathrm{pH}$-adjusted by the dropwise addition of $0.1 \mathrm{~mol} / \mathrm{L}$ sodium hydroxide solution. Under these unbuffered conditions, subsequent addition of polymer powder ( $0.15 \mathrm{wt} \%$ polymer) to the aqueous phase (at $35^{\circ} \mathrm{C}$ ) resulted in an immediate $\mathrm{pH}$ drop from the initial value, as shown in Fig. 8(a). The largest $\mathrm{pH}$ changes can be seen for the initial $\mathrm{pH}$ range 8.5-9.5 from which it is suggested that the maximum $\Delta \mathrm{pH}$ value (i.e., initial $\mathrm{pH}$-final $\mathrm{pH}$ ) of $\sim 2.5$ will occur at an initial $\mathrm{pH}$ of $\sim 9.5$. Unbuffered $\mathrm{pH}$ solutions producing a sufficiently stable $\mathrm{pH}$ in the range 8.5-9.0 were difficult to obtain in our experimental system, although from Fig. 8(a) it is unlikely that these would produce $\Delta \mathrm{pH}$ values significantly in excess of the currently observed maximum value.

The rapid change in $\mathrm{pH}$ observed, even before the onset of particle dissolution, suggests the consumption of hydroxide ions presumably involving ionisation of exposed hydroxyl groups. Thus, in Fig. 8(b) is shown the relationship between consumed $\mathrm{OH}^{-}$ions (per unit mass of polymer powder) and the final $\mathrm{OH}^{-}$ion concentration, which has the form of a high-affinity isotherm.

Above an initial $\mathrm{pH}$ of $\sim 7.8$, it was found that the hydration process was too rapid to take samples at intermediate times for viscosity determination, and therefore only final values after 25 minutes were obtained under these $\mathrm{pH}$ conditions. Fig. 8(c) shows that the kinetic curve for an initial $\mathrm{pH}$ of 7.76 exhibits a comparatively short induction time (cf. Fig. 4 at lower $\mathrm{pH}$ ). On the other hand, the maximum viscosity data in Fig. 8(d) show no substantive dependence on final $\mathrm{pH}$ in the range 7-10, indicating an absence of ionic interactions. 

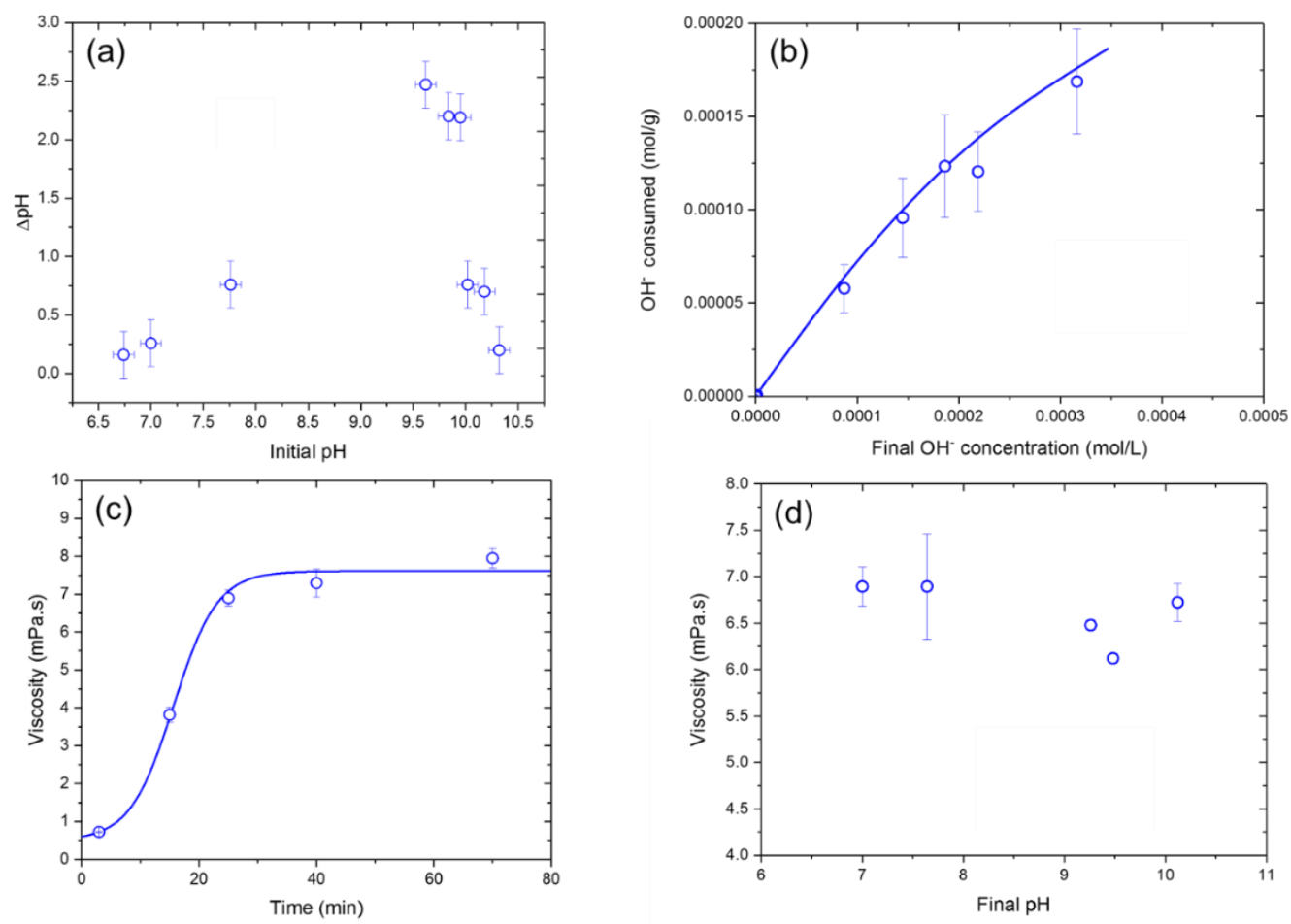

Fig. 8. (a) The effect of initial $\mathrm{pH}$ on $\mathrm{pH}$ change $(\Delta \mathrm{pH})$ at $35^{\circ} \mathrm{C}$ following the addition of $0.15 \mathrm{wt} \%$ EM 7000 FQ powder. (b) Isotherm $\left(35^{\circ} \mathrm{C}\right)$ showing the relationship between hydroxide ion consumed and final hydroxide ion concentration for EM $7000 \mathrm{FQ}$ powder. (c) Zero-shear viscosity-time profile for the hydration of $0.15 \mathrm{wt} \%$ polymer at $35{ }^{\circ} \mathrm{C}$ and initial $\mathrm{pH}$ 7.76. The curve drawn through the experimental data is based on Eq. (2) with $\eta_{0}(i)=0.48 \mathrm{mPa} \mathrm{s}, \eta_{0}(f)=7.61 \mathrm{mPa} \mathrm{s}, k=0.270 \mathrm{~min}^{-1}$, and $\tau_{\text {ind }}=15.7 \mathrm{~min}$. (d) Maximum zero-shear viscosity of polymer solutions as a function of final $\mathrm{pH}$ at $35^{\circ} \mathrm{C}$.

\subsection{Effect of ionic strength on hydration}

The properties of hydrophilic polymers in aqueous solution are often found to be influenced by the presence of salts [25,26], although in the present work there is the potential added complication of effects on crosslink breakage. Apparent viscosity-time profiles at $35{ }^{\circ} \mathrm{C}$ are shown in Fig. 9(a) for three sodium chloride concentrations. In these cases, the solution rheology of 1.0 and $2.5 \mathrm{~mol} / \mathrm{L} \mathrm{NaCl}$ solutions exhibited increased low-shear viscosity, 
compared with deionised water, preventing zero-shear viscosity estimation (cf. Fig. 2(a)). Instead, apparent viscosity values at $10 \mathrm{~s}^{-1}$ were used to follow the hydration kinetics. Again, these have been fitted using Eq. (2), providing the kinetic and viscosity parameters summarized in Table 3. Most apparent from the kinetic data is the steady increase in $\tau_{\text {ind }}$ and decrease in $k$ with increasing ionic strength up to $2.5 \mathrm{~mol} / \mathrm{L}$; thereafter, hydration is apparently completely inhibited at $5 \mathrm{~mol} / \mathrm{L} \mathrm{NaCl}$ (no viscosity increase was actually observed after 2 days), which is not to be expected if $k$ and $\tau_{\text {ind }}$ values are extrapolated from the lower $\mathrm{NaCl}$ concentrations. We conclude, therefore, that not only are the hemiacetal bond hydrolysis and polymer hydration rates lower in the presence of $\mathrm{NaCl}$, but $\eta_{o}(f)$ is also affected at higher $\mathrm{NaCl}$ concentrations for which the water activity, $a_{w}$, may be too low to participate in hydrogen bonding with polymer hydroxyl groups, necessary for hydration. This aspect has not been fully explored in the present study, but the results in Table 3 and Fig. 9(b) indicate that the rate constants decrease significantly with increasing ionic strength, and that there is a $\mathrm{NaCl}$ concentration region at 23.5-4 mol/L $\mathrm{NaCl}$ (i.e., corresponding to a water activity $a_{w} \sim 0.85$ [27]), below which hydration is critically slowed, or inhibited altogether, although the possibility of specific anion effects cannot also be ruled out, as found for the gelation of methylcellulose [26].

The increase in $\eta_{0}(f)$ at lower $\mathrm{NaCl}$ concentrations, evident from Fig. 9(a) and Table 3, is likely to be related to the viscosity of the aqueous $\mathrm{NaCl}$ solutions, as shown in Fig. 9(c), and which is consistent with literature data [28].

Table 3. Kinetic parameters for the hydration of $0.15 \mathrm{wt} \%$ EM $7000 \mathrm{FQ}$ in deionised water and $\mathrm{NaCl}$ solutions at $35^{\circ} \mathrm{C}$, based on viscosity data at a shear rate of $10 \mathrm{~s}^{-1}$.

\begin{tabular}{lllll}
\hline $\begin{array}{l}\mathrm{NaCl} \text { concentration } \\
(\mathrm{mol} / \mathrm{L})\end{array}$ & $\eta_{o}(i)(\mathrm{mPa} \mathrm{s})$ & $\eta_{o}(f)(\mathrm{mPa} \mathrm{s})$ & $\tau_{\text {ind }}(\mathrm{min})$ & $k\left(\mathrm{~min}^{-1}\right)$ \\
\hline 0 & 0.718 & 6.42 & 70.1 & 0.138 \\
1.0 & 0.762 & 7.38 & 101 & 0.0765 \\
2.5 & 0.893 & 7.65 & 180 & 0.0354 \\
5.0 & 1.20 & - & - & - \\
\hline
\end{tabular}



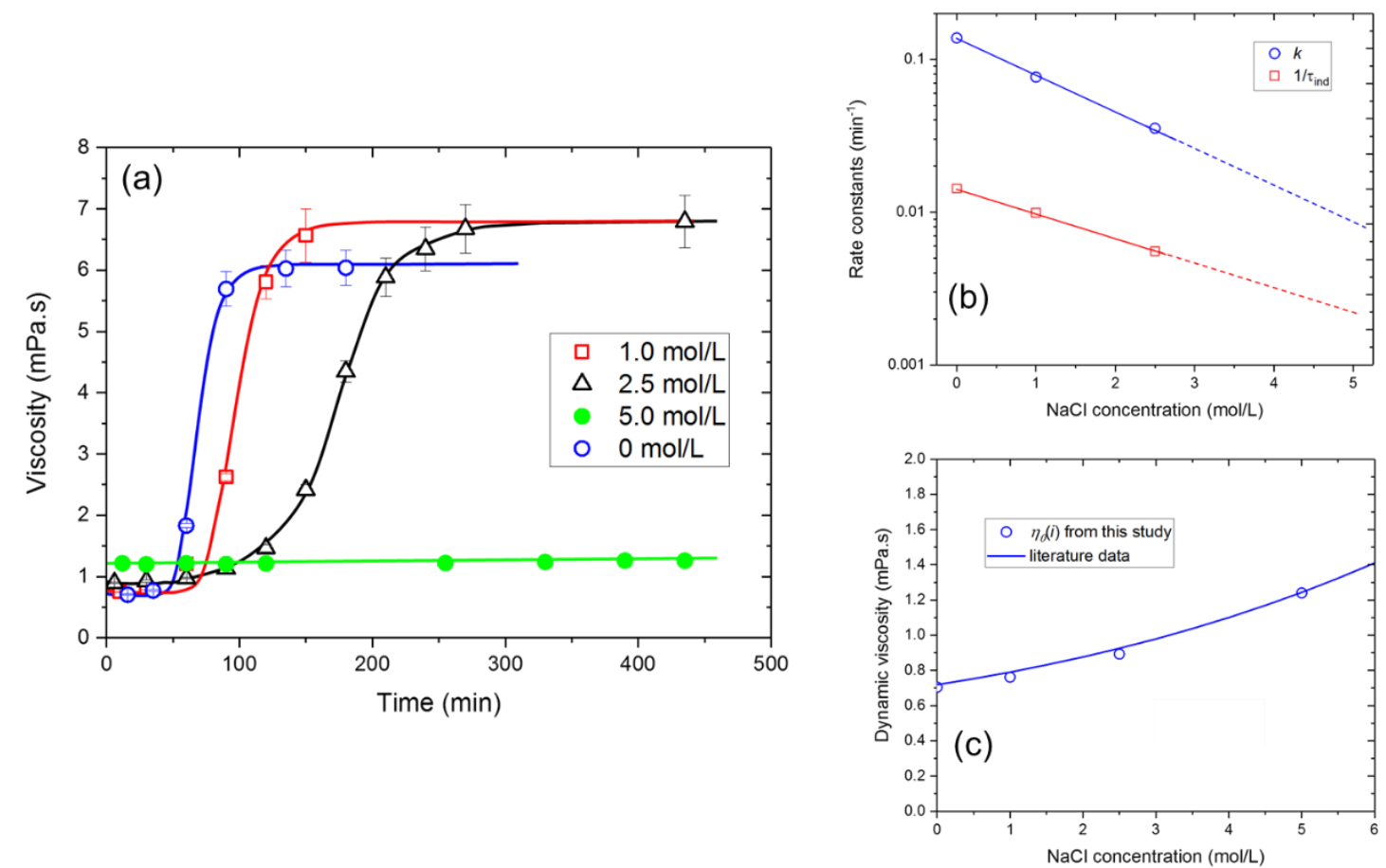

Fig. 9. (a) Effect of $\mathrm{NaCl}$ concentration on (a) apparent viscosity (at $10 \mathrm{~s}^{-1}$ )-time profiles for hydration of 0.15 wt\% EM $7000 \mathrm{FQ}$ at $35^{\circ} \mathrm{C}$; (b) hydration rate parameters calculated from the profiles in Fig. 9(a) using Eq. (2); (c) initial solution viscosity - points are $\eta_{0}(i)$ values and the drawn line is based on data from [28].

\subsection{Surfactant effects on hydration}

Over the years, significant emphasis has been given in the literature to the aqueous solution chemistry of polymers and surfactants and their interactions [29]. In the present study, we were also interested in determining the extent of any surfactant effects on the overall hydration of EM 7000 FQ.

Thus, in Fig. 10(a) are shown zero-shear viscosity-time profiles for polymer hydration in the presence of typical anionic, cationic and non-ionic surfactants, each at a concentration of $0.01 \mathrm{~mol} / \mathrm{L}$, which is above all their respective critical micelle concentrations. Once again, each profile has been fitted by Eq. (2), allowing the kinetic parameters to be determined (Table 4), from which it is evident that each surfactant solution leads to a decrease in the hydration rate 
constant, $k$ compared to deionised water. The induction times for SDS and TX-100 are similar to the deionised water value, although more significantly, CTAB appears to retard this process.

Also from Table 4, compared with deionised water it is seen that $0.01 \mathrm{~mol} / \mathrm{L} \mathrm{CTAB}$ and TX100 solutions are seen to generate $\sim 20 \%$ and $\sim 5 \%$ increases, respectively, in final solution zeroshear viscosity. This effect may be indicative of co-operative binding between the surfactant and polymer, with the formation of small micelle-like clusters involving the hydrophobic segments of the polymer, as proposed previously to explain the viscosity increase seen in the case of $1 \mathrm{wt} \%$ EHEC solutions in the presence of $\sim 0.01 \mathrm{~mol} / \mathrm{L} \mathrm{CTAB} \mathrm{[30].} \mathrm{The} \mathrm{clusters} \mathrm{act} \mathrm{to}$ create additional crosslinks between hydrophobic segments on different polymer chains, leading to an enhanced three-dimensional structure with increased viscosity, a situation which has also been treated theoretically and computationally [31].

However, the behaviour in the presence of the anionic surfactant SDS contrasts with that of the cationic and non-ionic surfactants, inasmuch as the viscosity attained for a concentration of $0.01 \mathrm{~mol} / \mathrm{L}$ is only $\sim 50 \%$ of the value for deionised water. In this case, it is suggested that interpolymer connectivity is hindered by the anionic micelles, a possibility also recognised theoretically [31]. This is suggestive of enhanced intra-polymer interactions (leading to coil shrinkage [32]) resulting from the adsorption of anionic surfactant micelles, which could electrostatically shield the hydrolysis of hemiacetal crosslinks. Intra-polymer interactions would also inhibit the hydration process by obstructing polymer chain disentanglement, thereby reducing the exposure to water molecules. 

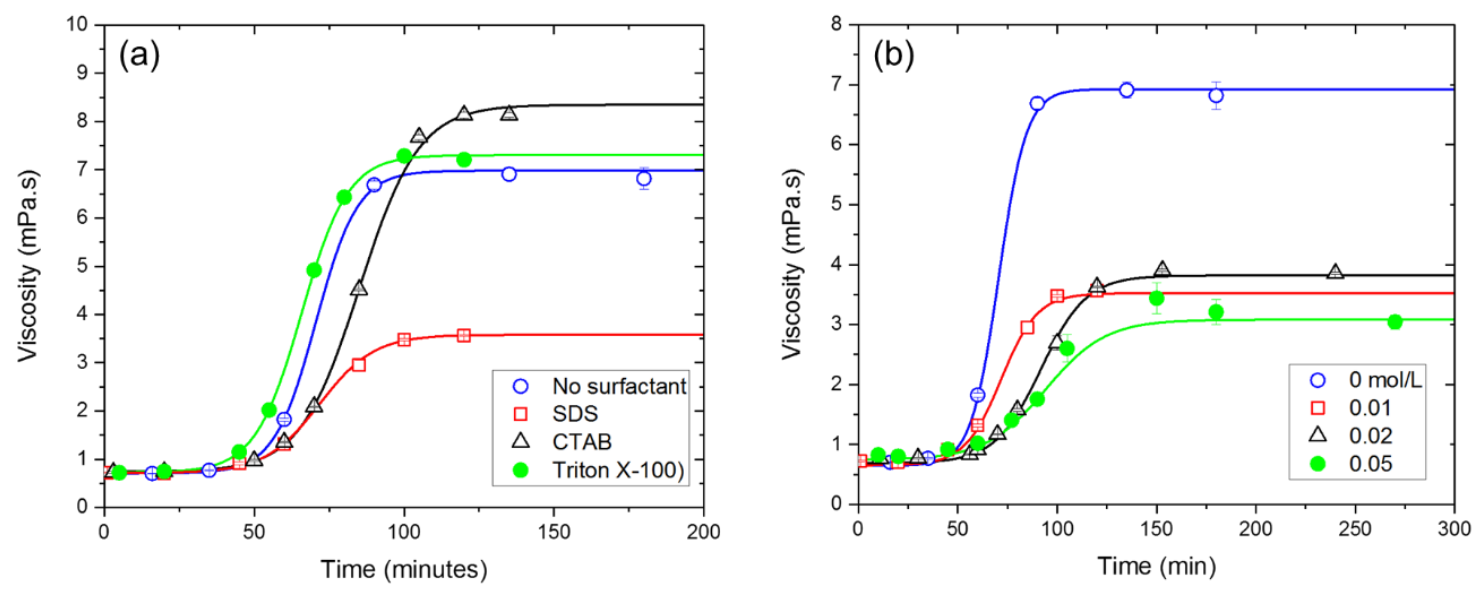

Fig. 10. Zero-shear viscosity-time profiles for hydration of EB7000 FQ polymer at $35^{\circ} \mathrm{C}$ : (a) aqueous $0.01 \mathrm{~mol} / \mathrm{L}$ surfactant solutions; (b) different SDS concentrations. Curves are fits to the data using Eq. (2).

Table 4. Kinetic parameters for the hydration of $0.15 \mathrm{wt} \% \mathrm{EM} 7000 \mathrm{FQ}$ in deionised water and $0.01 \mathrm{~mol} / \mathrm{L}$ surfactant solutions at $35^{\circ} \mathrm{C}$ based on zero-shear viscosity data and Eq. (2).

\begin{tabular}{lllll}
\hline $\begin{array}{l}\text { Surfactant } \\
\text { (concentration, } \\
\text { mol/L) }\end{array}$ & $\eta_{0}(i)(\mathrm{mPa} \mathrm{s})$ & $\eta_{o}(f)(\mathrm{mPa} \mathrm{s})$ & $\tau_{\text {ind }}(\mathrm{min})$ & $k\left(\mathrm{~min}^{-1}\right)$ \\
\hline None & 0.708 & & & 0.144 \\
CTAB $(0.01)$ & 0.745 & 6.98 & 70.6 & 0.101 \\
Triton X-100 (0.01) & 0.729 & 8.35 & 85.1 & 0.130 \\
SDS (0.01) & 0.714 & 7.31 & 65.8 & 0.111 \\
SDS $(0.02)$ & 0.751 & 3.58 & 71.8 & 0.0872 \\
SDS $(0.05)$ & 0.804 & 3.88 & 91.8 & 0.0663 \\
\hline
\end{tabular}

Fig. 10(b) shows that further increase in the SDS concentration causes only very slight changes in the hydration behaviour and the resultant solution viscosity. This suggests that the micellar clusters may have already attained their maximum size and created open, dilute structures, with a correspondingly minimum viscosity. For EHEC, Evertsson et al. also found that higher surfactant concentrations reduced the ability to undergo a sol-gel transition [32]. 
These findings indicate that as the number of polymer-bound surfactants on each chain increases, inter-polymer interactions are reduced.

\subsection{Significance}

Currently, much emphasis is being given to the development and applications of stimulusresponsive materials, which often involve polymers [33,34]. Most commonly, the stimuli investigated include temperature [34], $\mathrm{pH}$ [36-38] and ionic strength [36], and the applications span a number of technology sectors, from biomedical [39] to oil and gas [40].

From the results described above, it is clear that various factors can be used to effect control over the hydration behaviour of the glyoxal-crosslinked cellulose ether Bermocoll EM 7000 FQ. An analysis of the hydration process itself has been given in terms of two consecutive rate processes interpreted as involving the initial cleavage of the glyoxal crosslinks, followed by more rapid hydration of the polymer chains. High $\mathrm{pH}$ conditions promote base catalysis of the former reaction, thereby reducing the overall hydration time, since subsequent hydration of the polymer chains occurs rapidly under most conditions studied, the exception being for high ionic strength. A general equation formulated to describe the overall hydration process has allowed the effects of the different factors to be quantified. For a $0.15 \mathrm{wt} \%$ polymer concentration, for example, determining the effect of temperature on the viscosity-time profiles has allowed the estimation of activation energies for the crosslink cleavage and hydration reactions, as well as for viscous flow involving aqueous and hydrated polymer solutions.

Apart from the main influences of temperature and $\mathrm{pH}$, ionic strength $(\mathrm{NaCl})$ and surfactants have also been shown to influence the overall hydration process. The effects seen with surfactants are largely in agreement with theoretical predictions [31] and, based on the respective viscosities of the resulting polymer solutions, suggest different modes by which clustered surfactant micelles bind to the polymer. Thus, the cationic and non-ionic surfactants 
apparently augment inter-polymer interactions, whereas the anionic surfactant appears to favour intra-polymer interactions. These findings indicate that cationic (and possibly nonionic) surfactants are able to form bridges between neighbouring polymer chains which supplement the hydrophobic and hydrogen bonding responsible for the behaviour in deionised water. From Table 4 it can be seen that this results in $\sim 34 \%$ increase in viscosity in the presence of $0.01 \mathrm{~mol} / \mathrm{L} \mathrm{CTAB}, \sim 11 \%$ increase in viscosity for $0.01 \mathrm{~mol} / \mathrm{L} \mathrm{TX}-100$, and $\sim 40 \%$ decrease in viscosity for $0.01 \mathrm{~mol} / \mathrm{L}$ SDS.

\section{Conclusions}

Lightly surface-crosslinked polymer particles, such as Bermocoll EM 7000 FQ, were originally designed to hydrate slowly in order to facilitate more effective dispersion in water and prevent unwanted effects caused by rapid hydration. In the present paper we have considered various aspects of the more complex hydration of these crosslinked particles in aqueous solution.

Rapid cleavage of the crosslinks occurs at $\mathrm{pH}>\sim 8$, with relatively little effect on solution viscosity compared with lower $\mathrm{pH}$ conditions. Increased temperature also leads to more rapid hydration, and the viscosity exhibited by the polymer solutions is thermoreversible, decreasing with increasing temperature. The solution viscosity and hydration rates also decrease with increasing ionic strength up to a point where no hydration becomes evident (at $\sim 4 \mathrm{~mol} / \mathrm{L} \mathrm{NaCl}$ ).

The presence of surfactants was found to produce mixed effects on the polymer hydration behaviour, interpreted as resulting from their charge. Generally, $0.01 \mathrm{~mol} / \mathrm{L}$ solutions of all three surfactants produce slightly negative effects on hydration rate. The induction time is retarded by CTAB, with small effects for SDS and TX-100. Notably, however, compared to deionised water, final solution viscosity shows a significant increase for CTAB, while SDS produced $\sim 50 \%$ reduction. Such effects have been predicted in the past on theoretical grounds 
[31], and explained herein as reflecting inter-polymer micellar interactions for CTAB and to a lesser extent TX-100, and intra-polymer interactions for SDS.

\section{Acknowledgements}

The authors are grateful to the Department of Chemistry, University of Surrey and Quadrise Fuels International for supporting this work. 


\section{References}

[1] C. Clasen, W.-M. Kulicke, Determination of viscoelastic and rheo-optical material functions of water-soluble cellulose derivatives, Prog. Polymer Sci. 26 (2001) 18391919.

[2] P. Sharma, S.R. Modi, A.K. Bansal, Co-processing as a tool to improve aqueous dispersibility of cellulose ethers, Drug Dev. Ind. Pharm. 41 (2015) 1745-1758.

[3] E.I. Jullander, Methods of improving the dissolution of high-molecular substances, US Patent 2879268, 1959.

[4] J.H. Menkart, R.S. Allan, Readily dissolving cellulose derivatives and process therefore, US Patent 3072635, 1963.

[5] E.W. Hansen, K.H. Holm, D.M. Jahr, K. Olafsen, A. Stori, Reaction of poly(vinyl alcohol) and dialdehydes during gel formation probed by ${ }^{1} \mathrm{H}$ n.m.r. - a kinetic study, Polymer 38 (1997) 4863-4871.

[6] N. Sarkar, L.C. Walker, Hydration-dehydration properties of methylcellulose and hydroxypropylmethylcellulose, Carbohydrate Polymers 27 (1995) 177-185.

[7] A.R. Reid, Absorption rate of absorbent polymers by treating with glyoxal, US Patent $4051086,1977$.

[8] AkzoNobel Chemical Products $\quad$ literature https://celluloseethers.akzonobel.com/brands/bermocoll/, 2017 (accessed 8 November 2017).

[9] Y. Zhang, P.C. Zhu, D. Edgren, Crosslinking reaction of poly(vinyl alcohol) with glyoxal, J. Polymer Res. 17 (2010) 725-730.

[10] H.A. Waterman, C. Blom, H.J. Holterman, E.J. Gravenmade, J. Mellema, Rheological properties of human saliva, Arch. Oral Biol. 33 (1988) 589-596. 
[11] M.M. Cross, Rheology of non-Newtonian fluids: A new flow equation for pseudoplastic systems, J. Colloid Sci. 20 (1965) 417-437.

[12] H.A. Barnes, A Handbook of Elementary Rheology, University of Wales, Aberystwyth, 2000, p. 58.

[13] D. Risica, A. Barbetta, L. Vischetti, C. Cametti, M. Dentini, Rheological properties of guar and its methyl, hydroxypropyl and hydroxypropyl-methyl derivatives in semidilute and concentrated aqueous solutions, Polymer 51 (2010) 1972-1982.

[14] M. Rubinstein, R.H. Colby, Polymer Physics; Oxford University Press, 2003, pp. 367368.

[15] W.W. Graessley, Viscoelastic properties of entangled flexible polymers, Faraday Symp. Chem. Soc. 18 (1983) 7-27.

[16] C.G. Lopez, S.E. Rogers, R.H. Colby, P. Graham, J.T. Cabral, Structure of sodium carboxymethyl cellulose aqueous solutions: A SANS and rheology study, J. Polymer Sci. 53 (2015) 492-501.

[17] P. Kujawa, A. Audibert-Hayet, J. Selb, F. Candau, Rheological properties of multisticker associative polyelectrolytes in semidilute aqueous solutions, J. Polymer Sci. 42 (2004) 1640-1655.

[18] C.G. Lopez, R.H. Colby, P. Graham, J.T. Cabral, Viscosity and scaling of semiflexible polyelectrolyte NaCMC in aqueous salt solutions, Macromolecules 50 (2017) 332-338.

[19] C.K. Larsen, O. Gåserød, O. Smidsrød, A novel method for measuring hydration and dissolution kinetics of alginate powders, Carbohydrate Polymers 51 (2003) 125-134.

[20] R. Mills, Self-diffusion in normal and heavy water in the range $1-45^{\circ}$, J. Phys. Chem. 77 (1973) 685-688.

[21] H. Eyring, Viscosity, plasticity, and diffusion as examples of absolute reaction rates, J. Chem. Phys. 4 (1936) 283-291. 
[22] J. Kestin, M. Sokolov, W.A. Wakeman, Viscosity of liquid water in the range $-8{ }^{\circ} \mathrm{C}$ to $150{ }^{\circ} \mathrm{C}$, J. Phys. Chem. Ref. Data 7 (1978) 941-948.

[23] P. Sørensen, W.P. Jencks, Acid- and base-catalyzed decomposition of acetaldehyde hydrate and hemiacetals in aqueous solution, J. Amer. Chem. Soc. 109 (1987) 46754690.

[24] E. Romero-Nochebuena, J.A. Briones, M.G. Cupta, P.J. Brondsema, Additive composition for mortars, cements and joint compounds and cementitious compositions made therefrom, US Patent 8323400, 2012.

[25] E.B. Jørgensen, S. Hvidt, W. Brown, K. Schillén, Effects of salts on the micellization and gelation of a triblock copolymer studied by rheology and light scattering, et al, Macromolecules 30 (1997) 2355-2364.

[26] Y. Xu, C. Wang, K. C. Tam, L. Li, Salt-assisted and salt-suppressed sol-gel transitions of methylcellulose in water, Langmuir 20 (2004) 646-652.

[27] F. Chenlo, R. Moreira, G. Pereira, B. Bello, Kinematic viscosity and water activity of aqueous solutions of glycerol and sodium chloride, Eur. Food Res. Technol. 219 (2004) 403-408.

[28] J. Mazurkiewicz, P. Tomasik, J. Zapłotny, Relationships between water activity and viscosity of solutions, Food Hydrocolloids 15 (2001) 43-46.

[29] K. Holmberg, B. Jönsson, B. Kronberg, B. Lindman, Surfactants and Polymers in Aqueous Solution, second ed., John Wiley \& Sons, Ltd, 2003.

[30] A. Carlsson, G. Karlström, B. Lindman, Thermal gelation of nonionic cellulose ethers and ionic surfactants in water, Colloids Surfaces 47 (1990) 147-165.

[31] T. Furura, T. Koga, F. Tanaka, Effects of added surfactants on thermoreversible gelation of associating polymer solution, J. Polymer Sci. 42 (2004) 733-751. 
[32] H. Evertsson, S. Nilsson, Microviscosity in clusters of ethyl hydroxyethyl cellulose and sodium dodecyl sulfate formed in dilute aqueous solutions as determined with fluorescence probe techniques, Macromolecules 30 (1997) 2377-2385.

[33] B.R. Saunders, N. Laajam, E. Daly, S. Teow, X. Hu, R. Stepto, Microgels: From responsive polymer colloids to biomaterials, Adv. Colloid Interface Sci. 147-148 (2009) 251-262.

[34] D. Kuckling, Responsive hydrogel layers-from synthesis to applications, Colloid Polymer Sci. 287 (2009) 881-891.

[35] A.D. Drozdov, J. deClaville Christiansen, Review on the dynamics and micro structure of pH-responsive nano-colloidal systems, Adv. Colloid Interface Sci. 136 (2008) 2544.

[36] M. Sauer, W. Meier, Responsive nanocapsules, Chem. Commun. (2001) 55-56.

[37] Z. Dang, L. Liu, Y. Li, Y. Xiang, G. Guo, In situ and ex situ pH-responsive coatings with switchable wettability for controllable oil/water separation, ACS Appl. Mater. Interfaces 8 (2016) 31281-31288.

[38] B.H. Tan, K.C. Tam, Review on the dynamics and micro-structure of pH-responsive nano-colloidal systems, Adv. Colloid Interface Sci. 136 (2008) 25-44.

[39] C. de las Heras Alarcón, S. Pennadam, C. Alexander, Stimuli responsive polymers for biomedical applications, Chem. Soc. Rev. 34 (2005) 276-285.

[40] P.-F. Cao, J.D. Mangadlao, R.C. Advincula, Stimuli-responsive polymers and their potential applications in oil-gas industry, Polymer Rev. 55 (2015) 706-733. 
